\title{
Future electro-optical sensors and processing in urban operations
}

\author{
Christina Grönwall $^{1 \mathrm{a}}$, Piet B. Schwering ${ }^{\mathrm{b}}$,Jouni Rantakokko $^{\mathrm{a}}$, Koen W. Benoist ${ }^{\mathrm{b}}$, Rob A.W. Kemp ${ }^{\mathrm{b}}$, \\ Ove Steinvall $^{\mathrm{a}}$, Dietmar Letalick ${ }^{\mathrm{a}}$, Stefan Björkert ${ }^{\mathrm{a}}$ \\ ${ }^{\mathrm{a} S w e d i s h ~ D e f e n c e ~ R e s e a r c h ~ A g e n c y ~(F O I), ~ P O ~ B O X ~ 1165, ~} 58111$ Linköping, Sweden \\ ${ }^{\mathrm{b}}$ TNO, PO Box 96864, 2509 JG The Hague, The Netherlands
}

\begin{abstract}
In the electro-optical sensors and processing in urban operations (ESUO) study we pave the way for the European Defence Agency (EDA) group of Electro-Optics experts (IAP03) for a common understanding of the optimal distribution of processing functions between the different platforms. Combinations of local, distributed and centralized processing are proposed. In this way one can match processing functionality to the required power, and available communication systems data rates, to obtain the desired reaction times.

In the study, three priority scenarios were defined. For these scenarios, present-day and future sensors and signal processing technologies were studied. The priority scenarios were camp protection, patrol and house search. A method for analyzing information quality in single and multi-sensor systems has been applied. A method for estimating reaction times for transmission of data through the chain of command has been proposed and used. These methods are documented and can be used to modify scenarios, or be applied to other scenarios. Present day data processing is organized mainly locally. Very limited exchange of information with other platforms is present; this is performed mainly at a high information level. Main issues that arose from the analysis of present-day systems and methodology are the slow reaction time due to the limited field of view of present-day sensors and the lack of robust automated processing. Efficient handover schemes between wide and narrow field of view sensors may however reduce the delay times.

The main effort in the study was in forecasting the signal processing of EO-sensors in the next ten to twenty years. Distributed processing is proposed between hand-held and vehicle based sensors. This can be accompanied by cloud processing on board several vehicles. Additionally, to perform sensor fusion on sensor data originating from different platforms, and making full use of UAV imagery, a combination of distributed and centralized processing is essential. There is a central role for sensor fusion of heterogeneous sensors in future processing. The changes that occur in the urban operations of the future due to the application of these new technologies will be the improved quality of information, with shorter reaction time, and with lower operator load.
\end{abstract}

Keywords:Urban operations, EO-sensors, Signal processing forecast, Detection, Identification

\section{INTRODUCTION}

The European Defence Agency (EDA) is matching technological research needs to the required military. EDA is interested in specifying the military research needs for the coming ten years with a focus on enhancing the effectiveness in urban operations. Military and civil developments and investments in targeting electro-optical sensors and signal processing strategies need to be combined to enhance the needs for target recognition and identification in the urban environment. In complex urban operations a multitude of threats can be present while various neutral objects are present as well. With the increased power of EO sensors, both visible and infrared, more information becomes available for situational awareness. Data from self-defense imaging or alerting EO sensors of one platform may contain information of importance to other platforms. In particular, innon-line of sight operations, EO information from distributed sensor systems as well as different sources from different platforms are essential to improve situational awareness. However, the full potential of different EO sensors cannot be achieved on the operational field if their data are distributed around with no context.

The question is then how to connect the EO sensor data from different sensors, without overloading the communication systems and the operator. Normally, EO data streams require a communication system with high data rate. The data rate requirements can be reduced by processing data as early as possible for detection, classification and applying tracking

1hristina.gronwall@foi.se

Electro-Optical Remote Sensing, Photonic Technologies, and Applications VII; and Military Applications in Hyperspectral Imaging and High Spatial Resolution Sensing, edited by G. W. Kamerman, O. K. Steinvall, G. J. Bishop, J. D. Gonglewski, Proc. of SPIE Vol. 8897, 889702 - @ 2013 SPIE · CCC code: 0277-786X/13/\$18 - doi: 10.1117/12.2030412 
before sending it through the communication network. Currently there is no common understanding of an optimal distribution of processing functions between different platforms.

This problem was tackled by the EDA-funded study ESUO (Electro-Optical Sensors in Urban Operations ${ }^{2}$ ), which was performed 2011-2013 and reported in Ref. 1-2.The central theme of this study was to match the processing requirement of all the available sensors and platforms in a certain operation area with the available processing hardware and data transfer possibilities, in order to obtain optimum information at the right time and right location to the forces in this operating area. Combinations of local, distributed and centralized processing schemes were proposed. In this way one can match processing functionality to the available power and data rates to obtain the short reaction times needed in urban operations.

\subsection{Problem statement}

The urban environment distinguishes from other environments by extensive occurrence of

- occlusion of threats and friendly forces,

- short range detection and the accompanying short reaction time,

- strong perspective changes,

- heavy shadow and accompanying temperature shadow effects,

- 3D (area and height) danger zone.

In urban operation, the presence of high-risk threats will determine our ways of operation and the task we execute. These are:

- Self-defense, against ambushes, forces us to operate with closed hatch with very limited human vision,

- Situational awareness, $2 \pi$-hemispherical vision systems, requiring many detectors,

- Time for data processing, to be minimized to real-time, requiring automated detection and recognition data processing, also distributed over different platforms.

\subsection{Study approach}

Within the ESUO study, the following topics were investigated:

- Selection of priority urban scenarios,

- State-of-the art of EO-sensor, signal processing, data transmission and platform related issues,

- Forecast EO-sensor, signal processing, data transmission and platform related issues,

- Research topics and roadmap.

In the study three priority scenarios were defined. For these scenarios, present-day and future electro-optical sensors and associated signal/image processing approaches were studied. The priority scenarios were

- Camp protection (inside an urban area, i.e., dense populated area),

- Patrol with dismounted soldiers, ground vehicles and UAV,

- Search of a small house.

In this paper we present the evaluation method used for analyzing the processing and transfer of sensor data (Section 2). Based on the general evaluation and especially the scenario dependent analysis (Section 3), we forecast data processing, data transfer systems and platforms that will be in use in 10-20 years' time(Section 3). In Section 4, we present the most promising techniques that we have identified regarding EO sensors, data processing, data transfer and platforms. Identified research topics are listed in Section 5 and in Section 6 the general conclusions from the ESUO study are presented.

${ }^{2}$ EDA contract No 11.R\&T.OP.99. 


\section{EVALUATION METHOD}

Initially in the study, three scenarios were selected from a list of nine; the selection was performed based on feedback from the IAP3 group. For each of the selected scenarios several processing and transfer tasks were identified. E.g., processing of visual data collected by an UAV and then transfer of images or processed data to the commanding officer (CO). Based on these scenarios and tasks, we focussed our evaluation on parts of the scenarios where the data processing and transmission were likely to be challenging. From that evaluation, research topics relevant to defence applications in urban operations were identified. Thereafter, EO sensors, data processing, data transfer and EO-issues related to platforms that are relevant for today's urban operations were identified. We also predicted what would be in operational use in urban areas in 10-20 years. The data processing, data transfer and platforms-related issues were evaluated and compared according to the approach described in this section.

The flow of information is traced over the different steps in the communication channels from sensor platform to assess reaction time and quality of information (QI). Variables which influence reaction time and image quality are the platform locations, sensors, signal processing and data transfer possibilities. The schedule of the evaluation procedure is presented in Figure 1, starting with the information from the threat description. The potential presence of distributed processing is provided by the different processing blocks. In principle multiple sensors can observe the threat. In each of the functional blocks reaction time and image quality is modified according to the technical criteria of the different blocks. During the evaluation the scene is static. The evaluation of the task is ranked on the impact on the quantitative Reaction Time and Quality of Information, while the Cost and Vulnerability is used to constrain the ranking. Ranking of the different options is performed using a non-exhaustive multi-criteria analysis. Standardization of the criteria is performed making use of the minimum (set to zero) and maximum (set to one) limits; this will normalize the four different criteria. The four criteria were weighted together to produce the scoring to obtain the rank. The criteria are described further below.

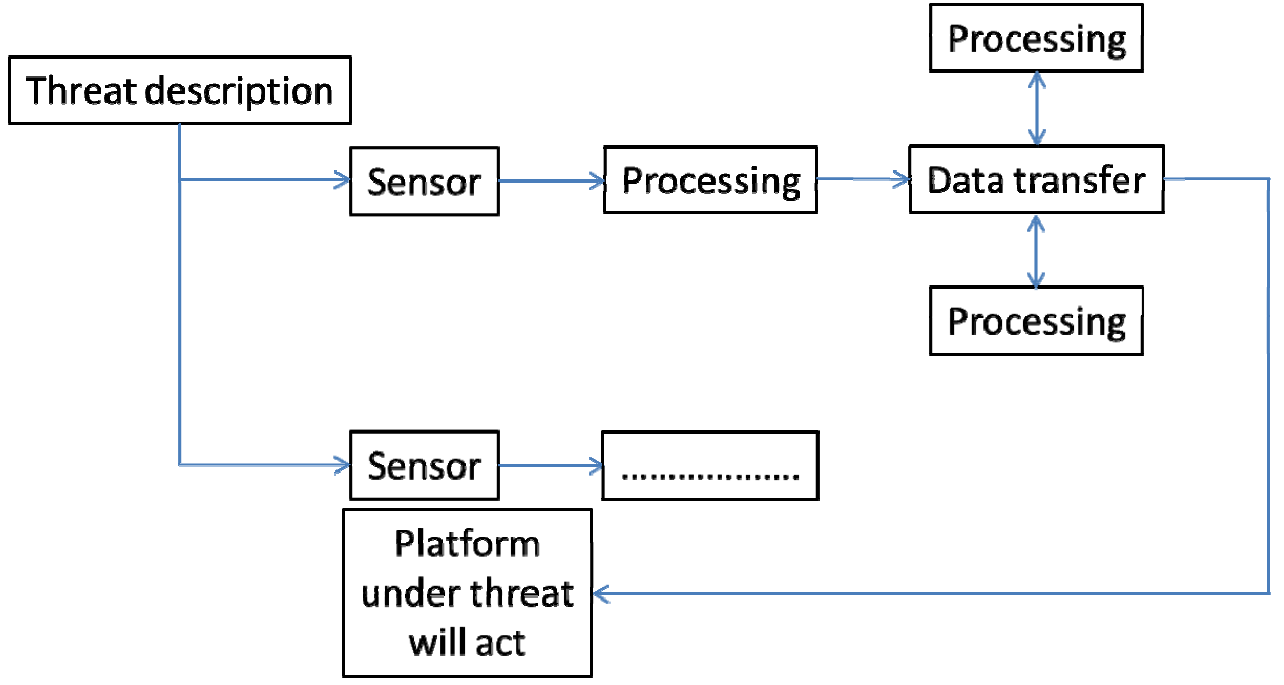

Figure 1.Overview of functional blocks in the evaluation procedure.

\subsection{Reaction time}

The reaction time is the time that passes between the first presence of the threat in urban scene, hence observable to individual sensors, and the availability of the information that the threat is detected at the acting platform operator (or commanding officer, $\mathrm{CO}$ ). The acting platform is the same as the platform under threat. This reaction time is calculated in each of the sensor, processing and data transfer blocks. In the analysis, the data set from the sensor that performs the data collection was followed upwards the chain of command until the (processed) data set has reached a commanding officer. Depending on the scenario/task, the $\mathrm{CO}$ can be placed in a vehicle, a local command post or on a C4I processing site. The possible locations for $\mathrm{CO}$ and for data processing are shown in Figure 2.

The processing of data can be performed by either a human or a machine and therefore reaction times for both human and automatic target search and identification were used. For calculating the reaction time for an (human) operator we require the search and act times. In complex environments with high clutter these time are larger than in simpler envi- 
ronments with less clutter (see for instance Ref.5). In Ref.4 quantitative testing has been performed on search strategies and these were applied in this study.

For automatic processing we use examples of references and gathered our experience. The initial delay for image enhancement, start-up of compression and further data transmission is set to $0.1 \mathrm{~s}$ (compared to $2 \mathrm{~s}$ for an operator). The search for targets in a field of view (FOV) not containing targets takes 0.03-10 s for each FOV depending on the processing times (compared to $5.7 \mathrm{~s}$ for an operator). The latency times given for automatic target detection and image fusion are used as search times, which implicate that image fusion is always performed. Some latency times are quite long (5-10 seconds) due to limited computational resources, for example when processing is performed on-board UAVs or in handheld equipment. For the FOVs containing targets, the latency times for target classification and data fusion are used, and they are usually 5-10 s/FOV (compared to $5 \mathrm{~s} /$ FOVfor an operator).For many vehicle and handheld sensors, the field of regard is larger than the FOV meaning that multiple FOVs have to be searched for targets.

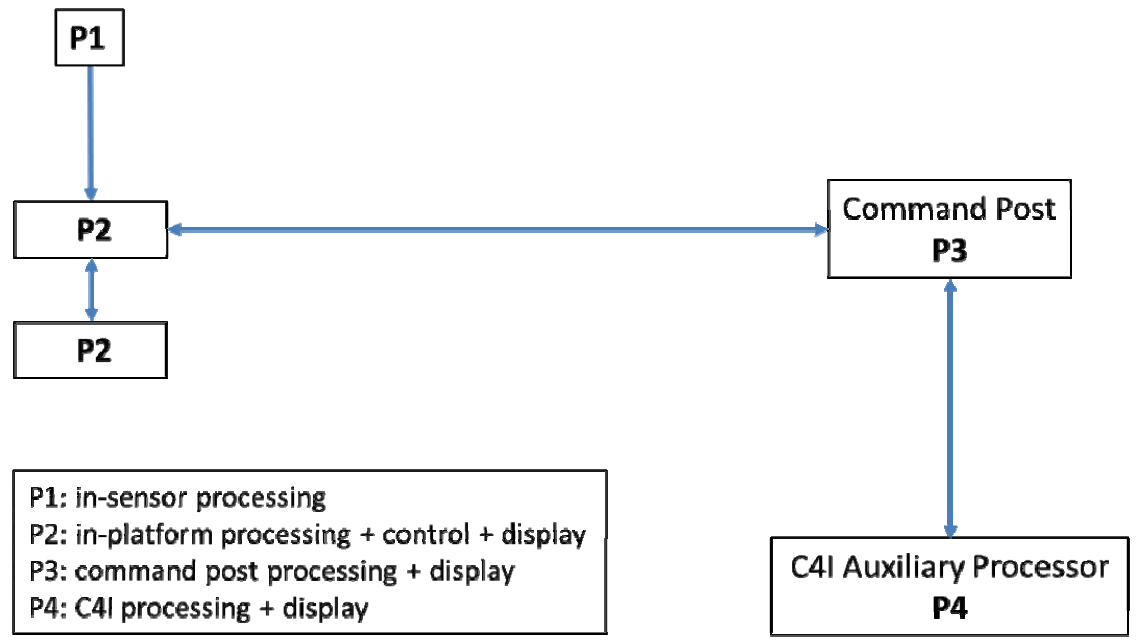

Figure 2. Signal processing locations P1-P4.

\subsection{Quality of information}

This provides the information quality of the present threat in the sensor scene to the platform operator (or CO). The increase in information quality is calculated for each of the sensor, processing and data transfer blocks.A well-defined approach for evaluation of performance of cameras existing of modern 2D-matrix arrays, is to use the Triangle Orientation Discrimination (TOD; Ref.6 and Ref.7). This method has several benefits over classical approaches as Minimum Resolvable Contrast (MRC) and Minimum Resolvable Temperature Difference(MRTD), see Ref.3. However, in general the availability of MRC, MRTD and TOD are unavailable for present-day state-of-the-art sensors. We therefore apply the approach to calculate the contrast and resolution of the sensor over the target with respect to the background.Resolution is given in the number of line pairs over the most relevant dimension of the threat,according to Ref.3, we require 1 line pair for detection, 3 for recognition and 6 for identification.

As we aim to rank the processing, the actual value is less important (note that these also depend strongly on the defined scenario). The approach described by Harney in Ref.8, based on calculation of bits of information,is designed for comparing information from different types of sensors and is used in this study. It follows a derivation from the line pairs defined in Ref.3, describing the number of require resolution elements (resels) and bits of information on the target. The advantage of this approach is that sensors making use of different subsets of the modalities intensity, angle-angle, range, color and velocity information (see Table 1) can be compared. The relation between the Harney metrics and the wellknown Johnson's criteria (Ref.3) is defined in Ref.8, and the Johnson criteria is used to calculated the lower limit on QI for performing a certain task. For all sensor data, with a Signal to Noise Ratio (SNR) equal to 2.8, it is required H=14.5 bits for detection, $\mathrm{H}=86$ bits for recognition and $\mathrm{H}=304$ bits for identification. As this work focus on EO-sensors and (distributed) data processing, the human eye itself is not analyzed. In the scenarios thisis taken into account to assess realistic sensor application. Its limitation in resolution is 0.33 milliradian (mr), with an FOV of 120 degrees. 
Table 1: Information content from various types of electro-optical sensors (see Ref.8for details).

\begin{tabular}{|c|c|c|c|c|c|c|}
\hline Sensor & Informati & on content in & the data & & & Model \\
\hline NVG & intensity & angle-angle & & & & $\mathrm{H}_{1}$ \\
\hline TIR & intensity & angle-angle & & & & $\mathrm{H}_{l}$ \\
\hline VIS & intensity & angle-angle & & colour & & $\mathrm{H}_{1}+\mathrm{H}_{\mathrm{H}}$ \\
\hline LRF & & & range & & velocity & $\mathrm{H}_{\mathrm{AR}}+\mathrm{H}_{\mathrm{M}}$ \\
\hline optical augmentation & intensity & angle-angle & range & & & $\mathrm{H}_{1}+\mathrm{H}_{\mathrm{AR}}$ \\
\hline IRST & intensity & angle-angle & & & & $H_{1}$ \\
\hline GV & intensity & angle-angle & range & & & $\mathrm{H}_{1}+\mathrm{H}_{\mathrm{AR}}$ \\
\hline 3D-imaging laser & intensity & angle-angle & range & & & $\mathrm{H}_{1}+\mathrm{H}_{\mathrm{AR}}$ \\
\hline SWIR & intensity & angle-angle & & & & $\mathrm{H}_{1}$ \\
\hline NIR & intensity & angle-angle & & & & $\mathrm{H}_{1}$ \\
\hline lidar scanner & intensity & angle-angle & range & & & $\mathrm{H}_{1}+\mathrm{H}_{\mathrm{AR}}$ \\
\hline Active VIS & intensity & angle-angle & range & & & $\mathrm{H}_{1}+\mathrm{H}_{\mathrm{AR}}$ \\
\hline
\end{tabular}

\subsection{Vulnerability}

For vulnerability different aspects of the components was checked with regard to the risk for degradation (hardware, disturbance levels). The level in which the vulnerability was addressed is:

- L (low): can withstand heavy hit, high disturbances, high interference levels (Electro Magnetic Compatibility EMC), high mobility etc.,

- $\mathrm{M}$ (medium),

- H (high): vulnerable; easy disrupted, cannot withstand low motion, low EMC, etc.

The vulnerability issues were analyzed for the technology areas sensors, signal processing and data transfer.

\subsection{Cost}

Regarding cost, the price range of the system components was estimated. The following cost ranges were addressed::

- L (low) around 10 kEuro (e.g. 1 uncooled thermal infrared (TIR) sensor),

- $\mathrm{M}$ (medium) around 100 kEuro (e.g. 1 cooled TIR sensor),

- H (high) around 1 MEuro (e.g. 1 advanced Infrared Search and Track (IRST) sensor).

Estimated prices are the cost to buy one component, development costs are not considered here. The cost issues were provided for the technology area sensors, signal processing and data transfer. Life-cycle costing was outside the scope of the study. As with sensors, all new components wereassigned a cost in this way. For processing we estimated the cost to be typically $10 \%$ of the sensor cost.

\section{SCENARIO DEPENDENT ANALYSIS AND EVALUATION}

A scenario dependent analysis is carried out by breaking down the processing distribution locations for each of the three prioritized scenarios, see example in Figure 3. Data processing and transmission cases are selected from the scenarios. They are selected on the assumption that the sensors are supporting the soldier and/or commander. The evaluation is based on assessing the reaction times and quality of information.

We analyze and evaluate the scenarios based on the capabilities for signal processing and transmission of sensor-based data. For each of the scenarios we select processing/transmission chains where we believed that the sensor data/information will support the soldiers and commanders. Threats are evaluated, while it is assumed that the subject under threat will act after a certain reaction time with the appropriate countermeasure. We assess the evaluation criteria for him to do so.

Even though our scenarios simplified the task of data processing/transfer in urban operations, the processing and transfer chain still were rather complex. To simplify the evaluation several assumptions were added: 
- It was assumed that the reaction time of the $\mathrm{CO}$ is negligible. In reality there will be some additional time delay in the decision making process,

- The data transmission hardware was selected to be fully supporting the required transmission data rates. This may overestimate the data rate,

- Other data and verbal communication would be over voice communication. There may be time delay and miscommunication associated with this. This may under estimate the reaction time at that link,

- Optical transmission conditions in the atmosphere were assumed to be one (optimal). As we were working at short range this was acceptable. Only some threats are observed at $1-2 \mathrm{~km}$ ranges and it only slightly overestimated the contrast of those threats,

- The mini-UAV or micro-UAV was operating in the right search areas.

We studied day and night capability, large search angles, comparison of active and passive imaging, line-of-sight capability, platform interoperability, processing function and their distribution. Some issues were analyzed for all three scenarios and some only for one scenario. In the analysis of future processing capabilities we focused on specific future processing technologies that provide operational benefits for the evaluation criteria reaction time and quality of information.For example, we studied the combination of information from the top view created by the UAVs with the ground view generated by sensors carried by soldiers and vehicles, the difference between off-board and on-board processing on the UAV, and combinations of sensor image information from different platforms located at different aspect angles from a building. We also considered line of sight (LOS) limitation, such as with vehicles located around the corner. We also studied the application of compressed sensing for sensor in the visual band on-board the vehicle and fusion of imagery with 3D information of the construction, to detect hideouts.

Specific focus areas were defined within each of the scenarios. These areas were chosen because of the military relevance, forecasted processing, and their potential to

- Decrease reaction time,

- Maintain or improve quality of information, vulnerability and cost.

For each scenario we provided a table with evaluation criteria for the forecasted equipment in the scenario. The analysis is especially tuned to the focus areas. An example of evaluation result is shown in Table 2, in this case processing near the sensors (i.e., in the mast) gave lowest reaction times and highest ranking.

To check in the analysis the task performance, in the quality of information for detection capability QI=14.5 bits are required, for recognition $\mathrm{QI}=86$ and for identification $\mathrm{QI}=304$ bits. In this example all sensors have QI values larger than 304 bits and thus, target identification is possible. It is noted that reaction time (RT) strongly depends on the required search time, proportional to the search angle and inversely proportional to the sensor field of view.

Table 2.Example analysis for the camp protection scenario.Comparison of approaches for keeping control of the camp perimeter, especially the gate area. RT: reaction time, QI: quality of information, C: cost, V: vulnerability, RANK: weighted summation of the criteria, trg: targeting.

\begin{tabular}{|c|c|c|c|c|c|}
\hline Sensor & RT & QI & C & $\mathbf{V}$ & RANK \\
\hline & [s] & [bits] & {$[\mathrm{H} / \mathrm{M} / \mathrm{L}]$} & {$[\mathrm{H} / \mathrm{M} / \mathrm{L}]$} & {$[0-1]$} \\
\hline Tower-TIR & 125,10 & 379,21 & MLM & LLM & 0,833 \\
\hline mast IRST & 5,10 & 3678,97 & MLM & LLM & 0,993 \\
\hline mast IRST is cueing 3D active SWIR+TIR (trg), processing in mast & 10,20 & 191022,94 & HMM & MLM & 0,911 \\
\hline mast-2D/3D active SWIR+TIR (trg) & 100,10 & 187343,98 & HML & MLL & 0,792 \\
\hline vehicle-TIR (smaller IFOV), human search \& identification & 427,40 & 789613,53 & MLM & LLM & 0,600 \\
\hline vehicle-TIR (larger FOV), human search \& identification & 128,15 & 197420,23 & MLM & LLM & 0,829 \\
\hline
\end{tabular}




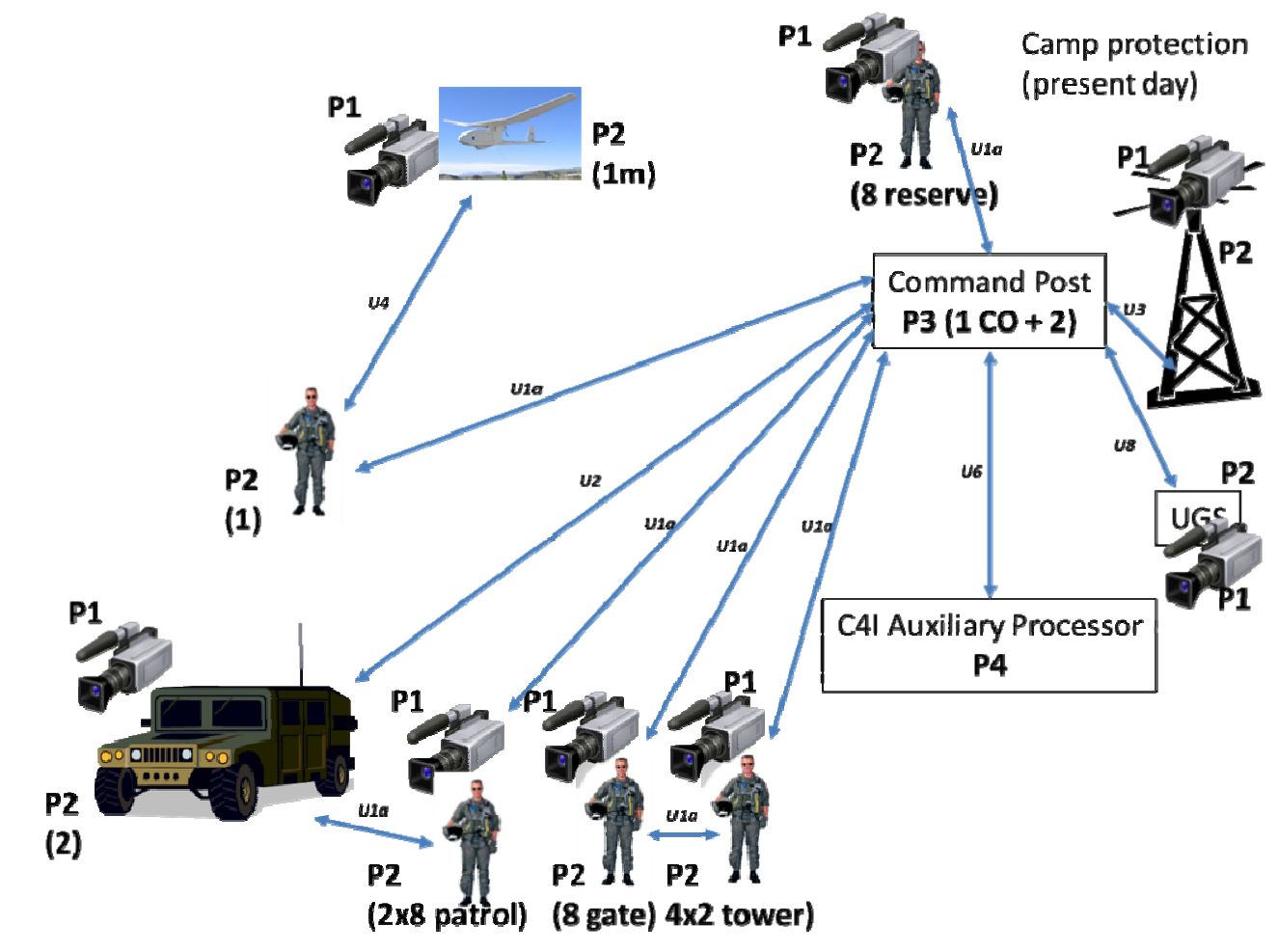

Figure 3. Signal processing locations and data transfer (use cases) for the Camp protection scenario (note that sensors are indicated with the camera symbol; although they can be TIR, or UGS sensors without cameras). The type of processing location (P1-P4) is noted and the lines between them illustrate data transfer.

\section{FORECASTS}

The forecast concerns autonomous and distributed processing (including hardware) of electro-optical sensor data that can be incorporated on land platforms within the next 10 to 20 years. Both passive and active sensors are predictedto be common in the future. This includes sensors with built-in advanced data processing, for example target detection, and sensor systems that operate at several wavelengths, which also can switch between passive and active modes. The sensors are the main supporting technology for the processing.Beside location for processing, the possibilities and challenges with fusion processing and graceful degradation of the information are described.

\subsection{Forecast processing}

We expect technical improvements of data processing hardware arising from increasing processing speed, smaller and cheaper processors and power reductions, as well as by combining different distributed processors at different locations. We also expect algorithm performance improvements, resulting in improved imagery and shorter reaction times, as well as the application of new techniques. Several local processing techniques will be enhanced versions of present-day techniques. This means they will be faster, because of increased processor configuration and speed, and will have higher quality of information due to increased algorithm complexity.

The forecast for new algorithm processing techniques is as follows:

- Compressed sensing (Ref.9). Application of these techniques may take more than 20 years to mature for operational use. Present-day quality of information is quite poor. Applications would clearly decrease the requirements on the data transfer system,

- Compression technology based on techniques like DSR (dynamic super resolution, Ref.10) will decrease required data rates, 
- Motion de-blurring and turbulence compensation will improve image quality, especially for imaging sensors on moving platforms. In particular, slow sensors such as uncooled TIR are affected by motion smear,

- Data fusion techniques will be common. There are clear benefits of fusing data from multiple heterogeneous sensors combined with intelligence sources over single-sensor operations in an urban environment. To protect operators from information overload, a fusion engine automatically tracks, fuses and relates observed objects to relevant (mission dependent) expected situations and potential threats. More fusion techniques will be developed, combining different sensors as well as sensor output with virtual 3D information,

- Algorithms for advanced image and data analysis will create high-level information. Examples are (semi-)automatic target characterization (fingerprinting) and detection of deviant behaviours,

- Application of sets of flexible high-power mini FPGA (Field Programmable Gate Array)processors. In this way, a few low power FPGAs can be applied on-board of simple, inexpensive cameras that can be distributed in large amounts. This paves the way for new system concepts combining data processing and sensor heads that are forecasted in different scenario,

- The optimal data collection from a scene usually means imaging from several aspect angles, i.e., moving the camera back and forth over the scene. These movements may not necessarily support the operator in his/her analysis task. To solve both the data processing and operator's need, new visualization schemes open for the possibility to show processed data in an order that is logical for a human. The key technologies concerns advanced data processing and visualization, see Ref.13.

\subsection{Forecast data transfer}

We also report trends that may affect the development of future military communication systems. The scarcity of available bandwidth limits the data rates for systems operating at lower frequencies. Larger bandwidths could be possible to obtain at high frequencies, using different sub-bands between 10 and $90 \mathrm{GHz}$, which could enable very high data rates. The high attenuation at these high frequencies limits the use of these systems mainly to LOS applications. When predicting the data rates for future military communication systems it is necessary to categorize the different systems, for instance according to:

- Line of sight (LOS) or non-line of sight (NLOS) conditions,

- Maximum range between units,

- Degree of mobility,

- Dedicated point-to-point communication system designed for large sensor data sets (e.g. real-time imaging data) or a communication system intended for heterogeneous data services (which often use multi-hop functionality).

The focus of the ESUO study was on urban operations and LOS is rarely available except at close distances or when using elevated platforms. However, even when considering the vehicle case it is not realistic to assume that e.g. three armoured fighting vehicles within a platoon will constantly be in LOS conditions.

We predict the special needs for future military communication systems. Several joint international projects are active which targets different applications. The research on soldier and vehicle communication systems is mainly focused on ad hoc network architectures with multi-hop functionality to increase range and availability. These systems can be seen as representative examples of what can be expected to be available operationally in a 10 to 15 year time-frame. These systems are not expected to provide data rates high enough for exchanging real-time video. However, for short ranges high data rates could be enabled in future military communication systems for the time frame in question. For instance, in LOS scenarios high frequency (e.g. $60 \mathrm{GHz}$ ) radio communications (Ref.11-12)or free-space optical communications (FSOC, Ref.13)are two technologies that are predicted to provide Gbps data rates also for mobile applications. The necessary technologies for providing short range NLOS radio-based data transfer are expected to be available for enabling real time video services within a local network (e.g. for a platoon of fighting vehicles). Possibly, the use of UAV's as relay nodes could be used to design a communication system where LOS conditions prevail. 


\subsection{Forecast platforms}

We also address the newly available platforms, as well as how platforms cooperate for improved situational awareness. Combinations of platforms are of interest as platforms sometimes have complementary information of an environment, and can improve their situational awareness by using additional data/information that cannot be gathered from the platform itself. Some platforms may be dedicated to carry sensors only, some carry processing/transmission equipment only, and some carry several or all parts. In our forecast for future platform operations, we therefore focused on the following subjects:

- Exchange of sensor information between vehicles observing the same target from different aspect angles. This improves the situational awareness of the local environment,

- Combination of sensor information on the ground with information recorded by UAV sensors from the air.

- Distributed processing of data to receive timely detection/classification results,

- Presentation of information, sensor image as well as meta-data, on 2D or 3D and on touch- displays. The latter also provide easy touch access,

- The Battle Management System (BMS) can be on-board of smaller platforms (squad level).

\section{PROMISING TECHNOLOGIES}

Based on the scenarios, we focussed our evaluation in the processing forecast on parts of the scenarios where the data processing and transmission are likely to be challenging. Based on that evaluation, research topics relevant to defence applications in urban operations were identified. In this section we summarize the most promising technologies in the most logical technology area; EO-sensors, processing, data transfer and platform.

\subsection{EO-sensors}

- For detection obtain WFOV EO-sensors to allow for faster search of large urban FOR areas,

- Automated processing (as in ATR and IRST) built-in into the sensors for fast cueing followed by visual inspection with high-resolution sensor for identification. Application of Multi-Function IRST,

- EB-CMOS as cheap high resolution alternative for night vision (Ref.14). This detector can be used in visible light and near-infrared bands to produce good signal-to-noise ratio in low light level conditions. In this way it can be a disruptive technology for passive and active night vision capability,

- Near infrared (NIR) or short wavelength infrared(SWIR) camera with gated viewing (GV) function laser for optical augmentation,

- Active SWIR systems for seeing around corner (Ref. 15)

- Active/passive SWIR with GV-function,

- 2D/3D active SWIR and TIR camera with GV function,

- 3D mapping based on laser, structured light (VIS) or simultaneous localization and mapping (SLAM, Ref.1718) processing of arbitrary imaging data.

\subsection{Data processing}

- Automatic search and detection to provide robust cues of locations of potential threats,

- Automatic post processing, i.e., after detection, such as clutter rejection in particular urban clutter (vehicles, buildings, persons) and robust recognition (classification techniques),

- Automatic determination and cueing for intent,

- Application of compressed sensing on-board the vehicle,

- Automatic processing in simple handheld sensors, 
- Motion de-blurring in vehicle- and handheld sensors,

- Turbulence compensation in long range identification systems,

- Fusion of several UAV sensor images (local on-board and distributed processing),

- Fusion of data from multiple heterogeneous sources (distributed processing),

- 3D reconstruction form various 3D sources: laser, structured light, stereo imaging,

- Fusion of imagery with 3D information of the construction,

- On-board distribution of processing in vehicle with flexible high-power mini FPGA, providing high performance cloud computing capability in network enabled operations; also providing fall-back capability to other signal processors,

- Automated processing (as in ATR and IRST) combined to the sensors for fast cueing followed by visual inspection with high-resolution sensor for identification,

- Image enhancement providing sharper imagery.

\subsection{Data transfer}

The development of a robust, high data rate communication system that supports real-time video transfer between vehicles is required in order to enable the optimal use of EO-sensors in the examined urban scenarios. The specified scenarios include both LOS and NLOS conditions, but the communication distances are generally quite short in this context. Current on-going development projects are not expected to fulfil this technology gap. However, enabling technologies are expected to be available which could enable the design of a local (e.g. platoon level) vehicle communication system with the primary aim of exchanging sensor data. Hence, in view of the findings in the ESUO study and taking into account the predictions concerning future data transfer possibilities, the proposed main priority concerning data transfer is the development of:

- A local communication network for real-time transfer of video and high-fidelity images between vehicles,

- Compression technologies to create improved image quality data, which also requires lower data rates, based on traditional compressing techniques like DSR or compressed sensing approaches.

\subsection{EO-issues related to platforms}

- Recce-vehicles with Multi-Function IRST,

- Camp mast equipped with similar automated detection processing on wide field of view (WFOV) EO sensors,

- On-board automated recognition,

- Human-machine interaction on-board to avoid overload of operators by selection of data and images to view,

- Battle management system on-board to address the right sensor and processing for the task(s) at hand.

\subsection{Remarks}

Some remarks on specific technologies where additional information is expected from other projects:

- Hyperspectral Imaging: Applications proposal require advice from EDA Cat B. DUCAS Project Arrangement No B 0294 IAP3 GC (expected end of 2013).

- SWIR Gated Viewing: Detailed technical results and roadmap will be provided by the EDA OB-Study 12.R\&T.OP.265 (expected summer 2013).

- Tactical Decision Aids: Roadmap results will be available from EDA OB study EOMOD 12.R\&T.OP.335 (in early 2014). 


\section{RESEARCH TOPICS}

Based on the evaluation of the scenarios, research topics relevant to the use of EO-sensors in military operations in urban terrain were identified. We also noted that EDA already have on-going or defined studies that will provide roadmap information on specific technical areas. The summary of the identified research topics is provided in Table 3 .

Table 3.Identified research topics.

\begin{tabular}{|c|c|c|c|}
\hline Technology & Objective & Research areas & TRL \\
\hline & & & at present \\
\hline & & & \\
\hline \multicolumn{4}{|l|}{ Sensors } \\
\hline WFOV sensors & Sensor for WFOV for detection & Large format IR detectors & 5 \\
\hline Multi-Function IRST & System for automated detection and human identification & Robust automated DRI processing, and vehicle motion & $6-7$ \\
\hline EB-CMOS & Cheap sensor for vision and detection & Low noise on large arrays, night illumination & 4 \\
\hline Active SWIR with GV-function & See around corner & Gate control & 3 \\
\hline $\begin{array}{l}\text { Active/passive SWIR with GV- } \\
\text { function }\end{array}$ & System for automated detection, recognition and tracking & Gate control and related target feature extraction & 6 \\
\hline 2D/3D active SWIR+TIR camera & System for automated detection, recognition and tracking & $\begin{array}{l}\text { High-resolution 3D detectors, related target feature } \\
\text { extraction }\end{array}$ & 6 \\
\hline $\begin{array}{l}\text { NIR/SWIR-camera + laser with GV- } \\
\text { function }\end{array}$ & Optical augmentation & Optics signature and false alarms & 6 \\
\hline \multicolumn{4}{|l|}{ Signal processing } \\
\hline Automated detection & Automated detection process & Selection of different processes & $7-8$ \\
\hline Post processing & Clutter rejection supporting automated detection processing & More robustness in high dynamic urban clutter & 3-4 \\
\hline Classification of intent & Classification of threats and intent & More robustness in high dynamic urban clutter & 3-4 \\
\hline Compressed sensing & Simplified sensor with lower data rate & Sufficient image resolution in real-time & 3 \\
\hline $\mathrm{HH}$ automated processing & Automated processing process in small hand held application & Small processing power, selection of cloud processing & 3 \\
\hline Motion deblurring & Motion deblurring in $\mathrm{HH}$ and moving platforms/vehicles & Interaction/feedback with platform stabilisation & $5-6$ \\
\hline Turbulence compensation & Improving resolution in long range path & High turbulence environments as warm roofs etc. & $5-6$ \\
\hline Fusion of imaging sources & Fusion of different UAV image frames from different wave bands & High data rate. UAV and multi vehicle based & $2-3$ \\
\hline Fusion heterogeneous sources & Data fusion from different sources of data & Limited asynchronous and heterogeneous data & $5-6$ \\
\hline $3 \mathrm{D}$ reconstruction & Reconstructing 3D from laser, stereo imaging and structured light & Reconstruction robustness from limited information & $3-5$ \\
\hline Fusion 3D & $\begin{array}{l}\text { Fusion of information from 3D/2D sensors with 3D construction } \\
\text { information }\end{array}$ & Robustness in fusion from limited scene information & 4 \\
\hline FPGA distribution & Cloud computing in Network Operated vehicle & Switch from local to cloud processing & $3-4$ \\
\hline $\begin{array}{l}\text { Fusion of data collected from } \\
\text { different perspectives }\end{array}$ & Increase perspective of the scene (ground-ground or air-ground) & Robustness in fusion from limited scene information & 4 \\
\hline $\begin{array}{l}\text { Automated cooperation of active } \\
\text { and passive imaging }\end{array}$ & Reduce operator workload. Increase target identification capability. & $\begin{array}{l}\text { Signal processing robust with/without active imaging. } \\
\text { Scheme how to use laser for optimal signal processing } \\
\text { result. }\end{array}$ & 3 \\
\hline \multicolumn{4}{|l|}{ Data transfer } \\
\hline Compression software & Dynamical resolution SW to increase decompressed image resolution & Sufficient video quality & 3-4 \\
\hline High data rate vehicle comm's & $\begin{array}{l}\text { Local communication network for real time transfer of video and high } \\
\text { fidelity images between vehicles }\end{array}$ & $\begin{array}{l}\text { UAV relay/multihop functionality for LOS comm's. } \\
\text { Robust NLOS radio system for small networks with } \\
\text { high spectral efficiency (incorporating e.g. adaptivity, } \\
\text { MIMO and DSA) }\end{array}$ & $5-6$ \\
\hline \multicolumn{4}{|l|}{ Platforms } \\
\hline Human Machine Interface & HMI to access multiple sensors without operator overload & Selection of data viewing & 6 \\
\hline Battle Management System & BMS managing sensor operation based on threat and operations & $\begin{array}{l}\text { Mapping threats to sensors, for best sensor selection } \\
\text { and persistent surveillance. }\end{array}$ & 7 \\
\hline
\end{tabular}




\section{SYNTHESIS AND CONCLUSIONS}

Within the Electro-Optical Sensors in Urban Operations (ESUO) study, the following topics are investigated:

- Selection of priority urban scenarios,

- State-of-the art of EO-sensor, signal processing, data transmission and platform related issues,

- Forecast EO-sensor, signal processing, data transmission and platform related issues,

- Research topics and roadmap.

Combinations of local, distributed and centralised processing are proposed. In this way, one can match processing functionality to the required power, and available data transfer, to obtain the right (which usually is very short in urban operations) reaction times. This work lists the most important research topics with roadmaps to support research and development. With the approach followed in ESUO, operations will benefit from shorter reaction times and higher quality of information. This is achieved by application of larger format sensors applied with automated processing. This will decrease the operator workload, as the operator does not have to visually inspect the entire scene, but can do only the final identification and/or decision step. Additional use of sensors that are presently in research and development stages will improve information content.

During this study, a method for analyzing information quality in single and multi-sensor systems has been identified and applied. A method for estimating reaction times for transmission of data through the chain of command has also been proposed and used. Distributed processing is proposed between hand-held and vehicle based sensors. This can be accompanied by cloud processing on board several vehicles. Additionally, for sensor fusion between different platforms and making full use of UAV-carried sensor imagery distributed and centralized processing is essential.

Operations will then depend on local processing in many cases. Robust local processing can be limited under conditions where the amount of data and complexity of threat information is large. In those cases, distributed processing can be addressed in nearby vehicles, as in cloud processing, to support the operations, providing robustness, and also to provide a backup processing. Local processing will form a graceful degradation of the distributed case.

While distributed processing is requested, imaging sensors require a high data rate transmission system. To keep fine details in the imagery, sufficient data rates are needed to be able to detect and recognise threats with automated processing. Several ways forward exist that can support this. Compressions techniques, such as compressed sensing and traditional compression methods can perform these tasks, while on the other hand the data rates of future data transfer systems may be extended.

Automated detection requires robust detection and sufficient post processing, so that the operator can be confident with the results from the processing. Especially in urban environments, it will take efforts to reject urban clutter in a robust manner, and be able to classify intent.

For identification purposes, image enhancement tools providing sharper imagery are supported by research into motion deblurring and turbulence compensation. Combination of various sources of information will improve quality of information in different scenarios.

The use of additional sensors also requires that an intuitive human machine interface will be implemented so that operator overload is avoided. Although automated processing supports this point additional sensors may give an additional load to the operator. The battle management system should support the operator to select the right sensors and processing for the task.

The priorities in urban operations for signal processing technology investment are in

- Robust automated detection for fast reaction times,

- Fusing information from different sources for increase of information quality.

The changes that occur in the urban operations of the future, due to the application of these new technologies, will be the improved quality of information, with shorter reaction time, and with lower operator load. The ESUO study suggested a roadmap for EDA with means to improve Europe's capabilities for urban operations and this is currently under discussion in the IAP3 group at EDA. 


\section{REFERENCES}

1. PES-ESUO-Publishable Executive Summary, Report to the European Defence Agency (EDA contract No 11.R\&T.OP.99), 2013, open access.

2. ESUO-WP1 - WP 5reports, Report to the European Defence Agency (EDA contract No 11.R\&T.OP.99), 2013, restricted.

3. Definition of nominal static range performance for thermal imaging systems, NATO AC/225-D/1096 STANAG 4337, edition 1, 25 August 1989.

4. Deaver, D.M., Moyer, S., Ra, C., "Modeling defined field of regard (FOR) search and detection in urban environments", SPIE Proc. Vol. 6207, 620802 (2006).

5. Neider, M.B., Zelinsky, G.J., "Cutting through the clutter: Searching for targets in evolving complex scenes", Journal of Vision Vol. 11(14): 7, pp1-16 (2011).

6. P. Bijl and J. M. Valeton, "TOD, the alternative to MRTD and MRC," Opt. Eng. 37(7), 1976-1983 (1998)

7. Piet Bijl, Jaap A. Beintema, Judith Dijk, Natasja van der Leden, "Effectiveness assessment of signal processing in the presence of smear", Optical Engineering 51(6), 063205 (June 2012).

8. Harney, R.C., "Information-based approach to performance estimation and requirements allocation in multisensory fusion for target recognition", Optical Engineering Vol. 36 (3), March 1997, pp789-798.

9. Willett RM, Marcia RF, Nichols JM; "Compressed sensing for practical optical imaging systems: a tutorial”, Opt. Eng. 50(7), 2011.

10. Judith Dijk, Klamer Schutte, Alexander Toet, Maarten Hogervorst, "Image enhancement on the INVIS integrated night vision surveillance and observation system”, Proc. of SPIE Vol. 7689, 2010.

11. http://www.mmwaves.com/products.cfm/product/20-194-0.htm

12. http://rf-circuits.info/radio/60-ghz-spectrum/

13. O. Gustafsson, F. Kullander, L. Sjöqvist, O. Steinvall, "Tactical free-space optical communication links - a study", FOI MEMO 3576, December 2011.

14. EOMAP IAP3-SRA-WP2-Progress Report-v0300, Report to the European Defence Agency, 29-09-2011

15. Endre Rapsi, Peter Lutzmann, Ove Steinvall, Magnus Elmqvist, Benjamin Göhler, GregorAnstett, "Advanced shortwavelength infrared range-gated imaging for ground applications in monostatic and bistatic configurations", Applied Optics, vol. 48, no. 33, p. 5956-5969, 2009, FOI-S--3221—SE.

16. P. Skoglar, U. Orguner, D. Törnqvist, and F. Gustafsson, "Road target search and tracking with gimballed vision sensors on a UAV", Remote sensing, 2012.

17. Durrant-Whyte, H. and Bailey, T., "Simultaneous Localization and Mapping (SLAM): Part I", IEEE Robotics \& Automation Magazine, 2(13), pp. 99-110, 2006.

18. Bailey, T. and Durrant-Whyte, H., "Simultaneous Localization and Mapping (SLAM): Part II", IEEE Robotics \& Automation Magazine, 3(13), pp. 108-117, 2006. 